\title{
La Phénoménologie de la vie religieuse du jeune Heidegger : une mise en perspective
}

Young Heidegger's Phenomenology of Religious Life: Broadening the Perspective La Fenomenología de la vida religiosa del joven Heidegger : una puesta en perspectiva

\section{Claudia Serban}

\section{(2) OpenEdition}

Journals

\section{Édition électronique}

URL : http://journals.openedition.org/assr/27125

DOI : $10.4000 /$ assr.27125

ISSN : $1777-5825$

Éditeur

Éditions de l'EHESS

\section{Édition imprimée}

Date de publication : 1 septembre 2015

Pagination : 213-230

ISBN : 9-782713224706

ISSN : 0335-5985

Référence électronique

Claudia Serban, «La Phénoménologie de la vie religieuse du jeune Heidegger : une mise en perspective », Archives de sciences sociales des religions [En ligne], 171 | 2015, mis en ligne le 01 septembre 2018, consulté le 01 mai 2019. URL : http://journals.openedition.org/assr/27125 ; DOI : 10.4000/assr.27125 


\section{Claudia Serban}

\section{La Phénoménologie de la vie religieuse du jeune Heidegger : une mise en perspective}

Le volume ${ }^{1}$ intitulé Phénoménologie de la vie religieuse (Martin Heidegger, Phénoménologie de la vie religieuse, trad. par Jean Greisch, Paris, Gallimard, 2011) est une traduction du tome 60 de l'édition complète des œuvres de Heidegger $^{2}$ - œuvres qui, selon la formule placée par l'auteur en exergue de ce projet de publication intégrale de ses travaux, sont à prendre plutôt comme des chemins: Wege, nicht Werke. Dans cette perspective, et sans nécessairement vouloir surestimer la métaphore du "chemin de pensée " de Heidegger (métaphore rendue classique par son commentateur Otto Pöggeler ${ }^{3}$ et alimentée comme on le voit par Heidegger lui-même), il est indispensable de situer ces contributions du jeune Heidegger à la philosophie de la religion à l'intérieur de son propre itinéraire philosophique ${ }^{4}$. Le volume qui nous intéresse principalement ici comprend deux cours et un projet de cours appartenant au premier enseignement fribourgeois de Heidegger (allant de 1919 à 1923, avant les quatre années passées à Marbourg en tant que professeur Extraordinarius de l'automne 1923 à l'été 1928 , et donc bien avant sa première grande publication de 1927 , Etre et temps,

1. Cet article représente une version remaniée et complétée d'un exposé fait à l'EHESS le 20 février 2013 dans le cadre du séminaire pluridisciplinaire "La théologie et les sciences sociales » organisé par Pierre Antoine Fabre et Dominique Iogna-Prat.

2. Martin Heidegger Gesamtausgabe, tome 60 : Phänomenologie des religiösen Lebens : 1. Einleitung in die Phänomenologie der Religion, 2. Augustinus und der Neuplatonismus, 3. Die philosophischen Grundlagen der mittelalterlichen Mystik, éd. par Matthias Jung, Thomas Regehly et Claudius Strube (noté GA 60), 1995, 2011².

3. Otto Pöggeler, Der Denkweg Martin Heideggers, Pfullingen, Neske, 1963 (La pensée de Martin Heidegger : un cheminement vers l'être, trad. par Marianna Simon, Paris, AubierMontaigne, 1967).

4. Nous ne pourrons pas tenir compte ici des éléments apportés par la publication des Schwarze Hefte, parus lorsque notre texte était déjà rédigé et qui, de toute façon, exigeraient une réflexion à part entière, que nous réservons pour des travaux à venir. Sur le rapport de Heidegger à la tradition juive, nous renvoyons à l'ouvrage désormais classique de Marlène Zarader : La dette impensée : Heidegger et l'héritage hébrä̈que, Paris, Seuil, 1990. Voir aussi le livre plus récent (et plus polémique) de Peter Trawny : Heidegger und der Mythos der jüdischen Weltverschwörung, Francfort, Klostermann, 2014 ; Heidegger et l'antisémitisme, trad. par Julia Christ et Jean-Claude Monod, Paris, Seuil, 2014. 
qui permit à Heidegger d'avoir un poste définitif à Fribourg). Il s'agit, en premier lieu, du cours du semestre d'hiver 1920-21, intitulé Introduction à la phénoménologie de la religion où, après un long préambule méthodologique, Heidegger propose une interprétation phénoménologique (nous verrons en détail ce que cela signifie dans ce contexte précis) de l'Épître aux Galates et des deux Épîtres aux Thessaloniciens de Saint Paul. Deuxièmement, nous trouvons dans ce même volume le cours de l'été 1921, Augustin et le néo-platonisme, qui contient notamment une interprétation (toujours phénoménologique) du livre $\mathrm{X}$ des Confessions. Enfin, le volume comprend aussi les notes préparatoires d'un cours projeté pour l'hiver 1918-19 qui, du fait des circonstances historiques liées à la fin de la Première Guerre mondiale, n'a pas pu être tenu, et dont le titre est très ambitieux : Les fondements philosophiques de la mystique médiévale ${ }^{5}$. Ce même cours a été annoncé par Heidegger pour le semestre d'hiver 1919-20, mais il a été finalement remplacé par un cours (qui forme le tome 58 de l'édition intégrale) intitulé Problèmes fondamentaux de la phénoménologie (cf. Theodore Kisiel, The Genesis of Heidegger's "Being and Time », Berkeley, University of California Press, 1995, p. 76).

Le premier enseignement fribourgeois de Heidegger, auquel ces cours appartiennent, est réuni le plus souvent sous la rubrique «herméneutique de la vie facticielle ${ }^{6}$ ». De fait, il s'agit d'une période où Heidegger est en dialogue, parmi ses contemporains proches, à la fois avec Husserl, Natorp, Jaspers, Troeltsch, mais aussi avec Dilthey, mort en 1911. Parmi ces derniers, c'est la double filiation husserlienne et diltheyenne qui mérite d'être soulignée et qui marque de son sceau la manière qu'a Heidegger de procéder en philosophie de la religion : d'une part, il propose une approche herméneutique attentive au caractère historique de ce qui est décrit et qui voit dans les écrits néo-testamentaires, patristiques et mystiques des expressions historiques de la vie du chrétien ; d'autre part, une approche phénoménologique, elle aussi attentive à la dimension vécue, mais en même temps plus soucieuse de la question méthodologique de l'accès à ce qui est à décrire, et plus portée vers des descriptions d'essence. La phénoménologie de la vie religieuse du jeune Heidegger est une phénoménologie herméneutique, ou encore une herméneutique phénoménologique, et c'est là que réside sans doute son

5. Au sujet de ces notes, nous renvoyons à l'étude minutieuse de Sylvain Camilleri : Phénoménologie de la religion et herméneutique théologique dans la pensée du jeune Heidegger, Dordrecht, Springer, 2008.

6. Pour sa réception en France, voir les travaux de Jean Greisch, dont notamment L'Arbre de vie et l'Arbre du savoir. Le chemin phénoménologique de l’herméneutique heideggérienne (1919-1923), Paris, Cerf, 2000, et de Sophie-Jan Arrien, dont surtout L'inquiétude de la pensée, Paris, PUF, coll. "Épiméthée ", 2014. L’option interprétative de Sophie-Jan Arrien, qui est de lire l'herméneutique de la facticité du jeune Heidegger pour elle-même et non comme un préambule à l'analytique existentiale déployée plus tard par Être et temps, nous semble particulièrement apte à saisir et à restituer l'originalité du projet de phénoménologie de la vie religieuse qui nous intéresse ici. 
principal intérêt pour la méthodologie des sciences religieuses. C'est un point sur lequel nous aurons l'occasion de revenir.

Mais pourquoi, on peut se demander, une phénoménologie de la vie religieuse ? Dans l'Allemagne de l'époque, cette conjonction de termes n'a rien d'évident. En philosophie, le courant phénoménologique est rattaché au nom d'Edmund Husserl, dont le premier ouvrage important est une Philosophie de l'arithmétique (1896) et le second, des Recherches logiques (1900-1901) élaborées dans un débat étroit avec la psychologie de l'époque. Husserl, juif converti au protestantisme, tout en n'étant pas complètement étranger aux questions théologiques ou religieuses (on dispose ainsi de plusieurs ouvrages sur son approche de la question de Dieu ${ }^{7}$ ), ne leur a jamais accordé une place centrale, ou même importante, dans sa propre phénoménologie. La position philosophique de Husserl, au tournant des années 10 , est celle d'un idéalisme transcendantal qui désactive la différence traditionnelle entre connaissance humaine et connaissance divine et entend fournir des descriptions eidétiques de l'expérience (de la perception, par exemple) qui sont censées valoir «même pour Dieu » - pris ici plutôt en tant qu'idée ou idéal, et non pas comme objet de la foi ou de la théologie. On ne trouvera donc pas, chez Husserl, quelque chose comme une phénoménologie de la vie religieuse ${ }^{8}$ même s'il produit par ailleurs de fines analyses des modalités de la croyance que l'on pourrait mettre avec profit au service d'une méthodologie des sciences religieuses.

Cependant, à Heidegger dont il connaissait bien l'appartenance au catholicisme, Husserl aurait « confié la tâche de jeter les bases d'une phénoménologie de la religion " ${ }^{9}$. Mais qu'est-ce qui prédestinait, pour ainsi dire, Heidegger à une telle tâche? Pour le comprendre, il est indispensable de faire un bref détour par quelques considérations biographiques.

\section{Un “ théo-logien " athée ?}

Fils du sacristain de l'église Saint-Martin de Messkirch (de sorte qu'un commentateur comme Dieter Thomä a pu écrire que « le vrai point de départ de l'histoire du texte heideggérien n'est pas la Forêt noire, mais l'élément catholique » (Dieter Thomä, Die Zeit des Selbst und die Zeit danach. Zur Kritik der Textgeschichte Martin Heideggers [1910-1976], Francfort, Suhrkamp, 1990, p. 35), Heidegger se destine initialement à des études de théologie. C'est son professeur de dogmatique, Carl Braig, qui contribue à éveiller son intérêt pour la philosophie, comme l'avait fait la découverte précoce du livre de Franz Brentano (professeur de

7. Voir surtout celui, plus récent, d'Emmanuel Housset : Husserl et l'idée de Dieu, Paris, Cerf Éditions, 2010.

8. Signalons toutefois le travail novateur mais malheureusement peu connu de l'élève strasbourgeois de Husserl, Jean Héring : Phénoménologie et philosophie religieuse, Paris, Alcan, 1926.

9. Comme le rappelle Jean Greisch dans son Avertissement du traducteur (Martin Heidegger, Phénoménologie de la vie religieuse, op. cit., p. 8). 
Husserl par ailleurs, qui apprécie en lui surtout l'auteur de la Psychologie descriptive d'un point de vue empirique) sur La diversité des acceptions de l'être d'après Aristote (1862), livre offert à Heidegger en 1907 par un ami de famille, Conrad Gröber, futur archevêque de Fribourg. Le parcours de Heidegger est marqué par l'interruption de ses études de théologie, suite à une crise de la foi catholique dont les premiers signes se manifestent dès 1914, même si en 1917 Husserl voyait encore en lui « un philosophe catholique avec de francs engagements confessionnels ${ }^{10}$ ». De cette crise de la foi, le témoignage le plus saisissant est une lettre du 9 janvier 1919 que Heidegger envoie au chanoine Engelbert Krebs, où il écrit : " le système du catholicisme m’est devenu problématique et inacceptable, mais non le christianisme ni la métaphysique (comprise en un sens nouveau)" (Apud Jean Greisch, Ontologie et temporalité, op. cit., p. 9). Dans cette affirmation s'annonce la rupture de Heidegger avec le catholicisme (due aussi, on le suppose, à l'influence de sa femme Elfride, épousée en 1917) et son rapprochement par rapport au protestantisme. Il faut souligner, en effet, qu'au moins jusqu'au milieu des années 20, malgré l'abandon de ses études de théologie et sa prise de distance radicale par rapport au catholicisme, Heidegger continue à baigner, intellectuellement et personnellement, dans l'élément du christianisme. Un témoignage célèbre en est une autre lettre, adressée par Heidegger à Karl Löwith (de huit ans son cadet, le futur auteur d'Histoire et salut - Weltgeschichte und Heilsgeschehen, 1953), doctorant à l'époque. Cette lettre fameuse, datée du 19 août 1921, mérite d'être citée ici puisqu'elle reflète l'identité philosophique de Heidegger à l'époque où il professait les cours que nous analysons et auxquels elle est seulement de quelques mois postérieure :

Je travaille concrètement, facticiellement à partir de mon « je suis »- à partir de ma provenance (Herkunft) spirituelle, facticielle comme telle - milieu - contextes de vie (Lebenszusammenhängen), à partir de ce qui m'est accessible en partant de là en tant qu'expérience vivante dans laquelle je vis. [...] C'est avec cette facticité de l'être-tel (Soseins-Faktizität), avec l'historique, que l'existence sévit (wütet); ce qui veut dire que je vis les obligations internes de ma facticité et je les vis aussi radicalement que je les comprends. - À cette facticité appartient - ce que je nomme ainsi brièvement que je suis un «théo-logien » chrétien. Cela implique un certain souci (Bekümmerung) de soi radical, une certaine scientificité radicale - une objectivité rigoureuse dans la facticité ; cela implique la conscience historique de «l'histoire spirituelle (geistesgeschichtlich)»- et je suis cela dans le contexte de vie de l'Université ${ }^{11}$.

La façon dont le philosophe allemand se définit ici comme «théo-logien chrétien » est à plus d'un titre remarquable. Certes, Heidegger écrit le mot théologien

10. Lettre à Natorp du 8 octobre 1917, citée par Jean Greisch, Ontologie et temporalité, Paris, Presses universitaires de France, 1994, p. 8.

11. Martin Heidegger, Lettre à Karl Löwith du 19 août 1921, in Zur philosophische Aktualität Heideggers, vol. II : Im Gespräch der Zeit, éd. par Dietrich Papenfuss et Otto Pöggeler, Francfort sur le Main, Klostermann, 1990, p. 29 ; trad. fr. (modifiée) par Guillaume Fagniez et Holger Nickisch, Archives de Philosophie, vol. 73, n 2/2010, p. 322-323. 
avec un tiret, en le décomposant selon son étymologie et en soulignant la composante qui correspond au logos : c'est à la manière de dire le divin plutôt qu'au divin lui-même comme objet de foi que semble faire référence cette auto-présentation. Cependant, pour définir son identité philosophique, Heidegger met l'accent ici sur sa facticité historique ou sa provenance concrète qui est, incontestablement, le milieu chrétien. Il semble ainsi formuler dans cette lettre une sorte de credo d'existence tout à fait semblable à celui qu'il repérera, par ailleurs, chez les premiers chrétiens : vivre radicalement dans la compréhension de sa propre situation historique.

Cela n'empêche pourtant que cette façon de s'auto-identifier à un théologien chrétien peut apparaître comme extrêmement fugace et passagère, et donc, rétrospectivement, extrêmement problématique, si l'on tient compte de l'évolution (même immédiate) de la position philosophique de Heidegger. Les cours de 1920 et 1921, sans contenir une prise de position expresse en matière de théologie ou de philosophie de la religion, reposent néanmoins sur une évidence cruciale pour le premier enseignement fribourgeois de Heidegger et donc pour ce qu'on appelle son herméneutique de la facticité : à savoir, ce qui apparaît dès son cours de l'hiver 1919-1920 comme une brève indication énigmatique, l'idée selon laquelle le christianisme serait le " paradigme historique le plus profond ${ }^{12}$ " pour l'exploration de la vie facticielle. Cela est d'une grande importance, pour autant que la facticité - concept clé du jeune Heidegger qui désigne la teneur concrète, historique de l'existence - ne se prête pas à quelque chose comme une description anhistorique. Comme nous pouvons le lire dans le même cours de l'hiver 1919-1920 : "le véritable organon de la compréhension de la vie est l'histoire (Geschichte), non pas en tant que science de l'histoire (Geschichtswissenschaft) [...], mais en tant que vie vécue "; autrement dit, "c'est dans l'histoire que réside le vrai "fil conducteur (Leitfaden)" pour des recherches phénoménologiques ", car « la vie effective et l'histoire sont [...] l'expérience directrice (Leiterfahrung) pour la recherche phénoménologique ${ }^{13}$ ». Par là, Heidegger s'inscrit ouvertement dans le sillage de Dilthey, que son maître Husserl avait cependant frappé du grave reproche d'historicisme par l'article publié en 1910 dans la revue Logos sous le titre «La philosophie comme science rigoureuse ${ }^{14}$ ». La phénoménologie

12. Martin Heidegger Gesamtausgabe, tome 58 : Grundprobleme der Phänomenologie, éd. par Hans-Helmuth Gander, Francfort-sur-le-Main, Klostermann, 1992, 2010² (noté GA 58), p. 61. Voir à ce sujet l'étude de Friedrich-Wilhelm von Herrmann : "Faktische Lebenserfahrung und urchristliche Religiosität. Heideggers phänomenologische Auslegung Paulinischer Briefe » (in Heidegger und die christliche Tradition. Annäherungen an ein schwieriges Thema, éd. par Norbert Fischer et Friedrich-Wilhelm von Herrmann, Hamburg, Meiner, 2007, p. 21-31) et celle de Sophie-Jan Arrien : "Foi et indication formelle. Heidegger, lecteur de saint Paul (19201921)" (in Le jeune Heidegger (1909-1926). Herméneutique, phénoménologie, théologie, éd. par Sophie-Jan Arrien et Sylvain Camilleri, Paris, Vrin, 2011, p. 155-172).

13. GA 58, p. 256, 246-247, 252, nous traduisons.

14. Voir Edmund Husserl, La philosophie comme science rigoureuse, trad. par Marc de Launay, Paris, Presses universitaires de France, 1989. 
herméneutique du jeune Heidegger revient au contraire, de manière explicite, à une réhabilitation de l'intérêt que présente l'histoire pour la recherche phénoménologique. Et l'histoire dont l'intérêt est souligné ici n'est pas, de prime abord, comme ce sera beaucoup le cas par la suite, l'histoire de la philosophie (ou encore, de la métaphysique), mais l'histoire des premiers chrétiens, qui fournit à elle seule le paradigme historique le plus profond pour une approche de la facticité.

Mais sur ce point encore, à regarder l'évolution immédiate de la pensée de Heidegger, telle qu'elle est reflétée dans ses cours, on pourrait avoir l'impression d'un diagnostic on ne peut plus éphémère. En effet, après ses cours sur Saint Paul et Augustin, Heidegger entame le premier de ses grands commentaires d'Aristote et on sait, par ailleurs, que l'ouvrage auquel il affirmait travailler pendant la première moitié des années vingt était un livre sur Aristote, un AristotelesBuch qui ne vit jamais le jour (car une autre inflexion, notons-le en passant, se fait voir dans son enseignement dans les années qu'il passe à Marbourg: la lecture de Kant, qui débouchera sur le Kantbuch de 1929, Kant et le problème de la métaphysique). Les premiers cours sur Aristote témoignent effectivement d'un tournant qui se manifeste aussi sous la forme virulente d'un plaidoyer en faveur d'un athéisme méthodologique qui s'exprime avec beaucoup de puissance :

La problématicité (Fraglichkeit) ne relève pas de la religion (ist nicht religiös), mais peut conduire pour la première fois dans la situation d'une décision religieuse. Je n'ai pas un comportement religieux en philosophant, même si je peux aussi comme philosophe être un homme religieux. [...] La philosophie doit, dans sa problématicité (Fraglichkeit) radicale et reposant sur soi, être principiellement a-théiste ${ }^{15}$.

Dans le même cours de l'hiver 1921-22, Heidegger écrit encore plus nettement au sujet de la philosophie qu'elle est athée lorsqu'elle se comprend radicalement ( $c f$. GA 61, p. 199), et semble récuser le rôle paradigmatique reconnu auparavant au christianisme, lorsqu'il affirme : "il ne faut aucune expérience religieuse pour que [l'] expérience soit vivante »(GA 61, p. 153). On peut considérer, bien sûr, que l'athéisme méthodologique de la philosophie, qui demeurera une constante dans la pensée de Heidegger, revient avant tout à dire qu'elle ne se confond pas et n'a pas à se confondre avec la théologie : elle n'a pas à se situer dans le registre de la foi religieuse. Telle est ainsi la neutralité théologique de Sein und Zeit, si l'on regarde par exemple la célèbre note (Martin Heidegger, Sein und Zeit, Tübingen, Niemeyer, 2001, p. 306) qui vise à assurer une certaine étanchéité entre ces analyses et ce qui peut sembler être leur correspondant du côté de la théologie chrétienne (par exemple, la doctrine du péché originel). Dans cette optique où l'athéisme méthodologique n'est rien de plus qu'une forme de neutralité, on peut sans contradiction élaborer une phénoménologie de la vie

15. Martin Heidegger Gesamtausgabe, tome 61 : Phänomenologische Interpretationen zu Aristoteles. Einführung in die phänomenologische Forschung, éd. par Walter Biemel et Käte Bröcker-Oltmanns, 1985, 1994² (noté GA 61), p. 197, nous traduisons. 
religieuse tout en adoptant une position d'athéisme méthodologique. Plus significatif et plus symptomatique nous semble cependant le décentrement incontestable de l'approche de la vie facticielle par rapport à ce qui était apparu comme son paradigme historique le plus profond - le christianisme - et par rapport à l'idée même d'expérience religieuse.

Est-ce à dire que Heidegger soit conduit à renier sa propre facticité historique, sa provenance chrétienne dont il faisait l'élément vital de son existence comme de son travail dans la lettre à Karl Löwith de 1921 que nous avons citée plus haut ? Qu'une telle interprétation serait trop hâtive et simpliste, c'est ce que prouve un aveu plus tardif (1937-38) qui va exactement dans le même sens que cette lettre, aveu que Didier Franck place au tout début de son livre Heidegger et le christianisme (Didier Franck, Heidegger et le christianisme, Paris, Presses universitaires de France, 2004) et qui est particulièrement instructif pour notre propos. Heidegger y note, à propos de son « chemin de pensée (Denkweg) » :

Et qui pourrait méconnaître que tout ce chemin s'accompagna silencieusement d'une explication (Auseinandersetzung) avec le christianisme - une explication qui n'est ni ne fut un "problème " rapporté mais le maintien (Wabrung) de la provenance (Herkunft) la plus propre - celle de la maison parentale, du pays natal et de la jeunesse - et simultanément (in einem) la séparation (Ablösung) douloureuse d'avec tout cela ? Seul qui fut ainsi enraciné dans un monde catholique effectivement vécu pourra pressentir quelque chose des nécessités auxquelles le chemin de mon questionnement fut soumis comme à des secousses telluriques souterraines (unterirdische Erdstösse). Les années marbourgeoises y ajoutèrent l'expérience plus directe d'un christianisme protestant mais déjà comme de tout ce qui devait être fondamentalement surmonté (überwunden) sans pour autant être détruit (zerstört).

Il ne convient de parler de cette explication la plus intime et qui ne porte pas sur des questions de dogmatique ou sur des articles de foi, mais uniquement sur la question de savoir si le dieu nous fuit ou non (ob der Gott vor uns auf der Flucht ist oder nicht), et si nous-mêmes nous pouvons encore véritablement, c'est-à-dire en tant que créateurs (als Schaffende) en faire l'expérience.

Et il ne s'agit pas non plus d'un simple arrière-fond "religieux » de la philosophie, mais de l'unique question de la vérité de l'être qui seule décide du "temps » et du "lieu» qui nous sont historialement impartis (aufbehalten) au sein de l'histoire de l'Occident et de ses dieux ${ }^{16}$.

Bien sûr, ici comme ailleurs, l'auto-interprétation de Heidegger ne fait pas loi. Elle nous encourage cependant à ne pas perdre de vue ce qui, dans les années 30 et par la suite, complique considérablement la position de Heidegger par rapport à la tradition (judéo -) chrétienne : la lecture de Hölderlin et celle de Nietzsche - autrement dit, la confrontation au double motif de la fuite des dieux et de la mort de Dieu -, ainsi que la présence de plus en plus marquée de la mythologie, de la poésie et de la philosophie présocratique grecques. Dès le

16. Martin Heidegger Gesamtausgabe, tome 66 : Besinnung, éd. par Friedrich-Wilhelm von Herrmann, Francfort, Klostermann, 1997, p. 415-416, apud Didier Franck, Heidegger et le christianisme, op. cit., p. 9, trad. modifiée. 
milieu des années 30 jusqu'au célèbre entretien de la revue Spiegel (1966) publié seulement après sa mort, la méditation philosophique de Heidegger est travaillée intérieurement, ou plutôt souterrainement, par la pensée (et l'attente) du « dernier dieu ${ }^{17}$ » qui "seul peut nous sauver ». Cette pensée du dernier dieu est dirigée manifestement contre le Dieu chrétien (et du fait de cette opposition qui se fait parfois très explicite ${ }^{18}$, l'on a pu identifier chez Heidegger des tendances, non seulement anti - [judéo -] chrétiennes, mais même néo-paganistes), pour autant qu'il s'agit d'un « dieu extrême auquel l'éternité est interdite ${ }^{19}$ ». Didier Franck souligne pour sa part (et c'est le fil conducteur de son ouvrage déjà mentionné) que l' « explication avec la métaphysique et le débat avec le christianisme appartiennent [...] au même chemin de pensée » (Didier Franck, Heidegger et le christianisme, op. cit., p. 10), en nous renvoyant implicitement au principe posé par la Lettre sur l'humanisme à Jean Beaufret (1947), selon lequel c'est seulement à partir de l'être que "peut être pensé et dit ce que doit nommer le mot "Dieu" " (GA 9, p. 351; trad. par Roger Munier in Questions III et IV, Paris, Gallimard, 1990, p. 112) - ce qui revient à subordonner la question de Dieu à la question de l'être ${ }^{20}$.

\section{La phénoménologie de la vie religieuse}

Il nous fallait ce long et sinueux détour avant d'aborder frontalement la Phénoménologie de la vie religieuse du jeune Heidegger, car celle-ci sortirait appauvrie et aplatie, nous semble-t-il, d'une lecture décontextualisée. Essayons à présent de préciser ce qui nous apparaît comme l'apport le plus original et le plus important des cours de 1920 et 1921 . La catégorie centrale de cette interprétation phénoménologique de Saint Paul et d'Augustin est celle que l'on trouve au cœur de l'explicitation de la facticité comme teneur concrète et vivante de l'existence : il s'agit de l'historicité (Geschichtlichkeit). Le fait que l'interprétation soit phénoménologique, et non pas simplement exégétique, motive en

17. Voir à ce sujet les analyses d'Otto Pöggeler dans Neue Wege mit Heidegger, Munich, Karl Alber, 1992, p. 465 sq.

18. Il suffit de mentionner à cet égard l'exergue de la section VII des Contributions à la philosophie intitulée "Le dernier dieu " : "Le tout autre contre ceux qui ont été, et surtout contre le chrétien (Der ganz Andere gegen die Gewesenen, zumal gegen den Christlichen)" (Martin Heidegger Gesamtausgabe, tome 65 : Beiträge zur Philosophie (Vom Ereignis), éd. par Friedrich-Wilhelm von Herrmann, Francfort, Klostermann, 1989, 1994², 2003³, p. 403).

19. Selon l'expression de Françoise Dastur dans La phénoménologie en questions, Paris, Vrin, 2004, p. 250.

20. Mentionnons en passant que c'est contre une telle subordination que s'est insurgée la phénoménologie française post-heideggérienne, depuis l'ouvrage publié par Levinas en 1974 : Autrement qu'être ou au-delà de l'essence, jusqu'au livre de Jean-Luc Marion, Dieu sans l'être, paru en 1982. Notons aussi la réaction d'esprit thomiste que ce dernier ouvrage a suscitée chez certains théologiens français (voir par exemple Dominique Dubarle, Dieu avec l'être: de Parménide à Saint Thomas : essai d'ontologie théologale, Paris, Beauchesne, 1986). 
outre la distinction cruciale de deux acceptions de l'histoire : l'histoire objective (Objektsgeschichte) et l'histoire d'accomplissement (Vollzugsgeschichte) (voir GA 60, p. 84, 88-89, 90 ; trad. fr., p. 95, 100, 101).

L'histoire objective est, comme son nom l'indique, celle qui a à faire à des objectivités : un texte, des mots, une certaine langue, qui s'éclairent à partir de certaines conditions historiques elles-mêmes objectives. Sa démarche consiste donc à tenir à distance le sens, à le considérer comme passé et à se rapporter à lui à la façon d'un spectateur désintéressé. L'histoire d'accomplissement s'efforce quant à elle de retrouver, par delà les significations objectives qui s'expriment dans le discours, l'expérience ou l'accomplissement concret et vécu de ces significations - ou la vie dont elles sont les expressions. Et ce n'est pas l'objectivation qui est dans cette perspective première, mais l'accomplissement (subjectif, on serait tenté de dire, ou personnel, voire interpersonnel, dans le cadre de la communauté des premiers chrétiens) qui la fonde. Adopter le point de vue de l'accomplissement, c'est aussi échanger la perspective du passé pour celle du présent, car il s'agit d'une épreuve qui peut être assumée et répétée d'une manière vivante, qui garde donc son actualité dans tous les sens du terme. L'histoire d'accomplissement, loin d'être l'œuvre d'un sujet confiné à une posture théorique, est intimement solidaire de l'historicité d'un homme ou d'un soi vivant, donc de l'histoire " que nous sommes nous-mêmes (das wir vielmehr selbst sind) »: il importe en effet de comprendre que ce "retour à l'historique (Rückgang in das Historische) " se fait " à travers l'histoire propre (durch die eigene Geschichte) (GA 9, p. 34 ; cf. GA 60, p. 173) ", qu'il y a donc une circulation entre le passé et le présent, entre la vie historique passée et la vie historique présente.

À l'origine de l'intérêt de Heidegger pour la figure de Saint Paul se trouve par ailleurs le fait même que celui-ci est parvenu au christianisme "à travers une expérience originaire (ursprüngliche Erfahrung) et non pas à partir d'une tradition historique (historische Tradition) (GA 60, p. 69 ; trad. fr. modifiée, p. 79) ». Cela fournit le principe même de la lecture phénoménologique des Épîtres, qui est de retrouver "l'accomplissement de la situation historique du phénomène (Vollzug der geschichtlichen Situation des Phänomens) (GA 60, p. 84 ; trad. fr., p. 95)» (en l'occurrence, l'expérience proto-chrétienne qui a été celle de Paul lui-même). C'est un point qui est souligné avec radicalité : "ce qui est visé, ce n'est pas l'idéal d'une construction théorique, mais l'originarité de l'absolument historique (Absolut-Historisch) dans son absolue irrépétabilité (Unwiederholbarkeit) (GA 60, p. 88 ; trad. fr. modifiée, p. 100) ». Il ne s'agit pas de revivre le passé, mais d'accomplir ou de vivre le présent avec la même radicalité qui a été celle de Paul devant sa propre situation historique.

L'historicité absolue et irrépétable est en effet ce qu'exprime le concept de situation, que Heidegger met en avant : l'unicité de la situation vient de ce qu'elle est vécue et accomplie, et la prise en compte de la situation ainsi déterminée est 
le ressort même qui fait passer de l'histoire objective à l'histoire d'accomplissement (cf. GA 60, p. 90 ; trad. fr., p. 101). Mais la situation des premiers chrétiens, dont l'éclaircissement guide l'interprétation des Épîtres de Paul, est en ellemême paradoxale, et ce paradoxe se lit dans la manière même dont ils infléchissent le sens du vocable grec parousia : ils n'y voient pas un événement présent, mais le retour futur du Christ, dont la présence est déjà, en un sens, de l'ordre du passé (cf. GA 60, p. 102 ; trad. fr., p. 114). Par là, le centre de gravité du temps se trouve déplacé du présent vers l'avenir. La situation se définit donc aussi à partir de sa temporalité spécifique. Pourtant, Heidegger refuse de voir dans le rapport qu'ont les premiers chrétiens à la parousie une simple attitude d'attente neutre : il insiste au contraire sur la charge affective de l'espérance chrétienne qui est par nature mêlée d'insécurité (Unsicherheit) (GA 60, p. 105 ; trad. fr., p. 117), insécurité qui est aussi inquiétude (Unruhe, absence de repos).

Cette insécurité de la vie chrétienne qu'implique sa situation tendue vers la parousie infléchit la manière dont elle est accomplie. Chez Paul, elle révèle une dimension de l'accomplissement qui est décisive pour comprendre la situation des premiers chrétiens : la négativité qui s'exprime par le vocable hôs mê (ou «comme non ${ }^{21}$ ») de 1 Cor. 7, 29-31: "que ceux qui ont des femmes soient comme n'ayant pas ", etc. Dans le hôs mê, Heidegger voit s'exprimer une modalité de l'accomplissement affectée par une négativité ou un "non » qui est "à la mesure de l'accomplissement (vollzugsmässiges Nicht) » (un "nonaccomplissant », comme traduit Jean Greisch) (GA 60, p. 109 ; trad. fr., p. 123), mais qui ne revient pas à désactiver l'accomplissement de la vie facticielle. Ce "non " est lui-même une coordonnée de la situation historique, donc une coordonnée de l'accomplissement lui-même.

Qu'est-ce qui détermine cet infléchissement de l'accomplissement de la vie facticielle chez les premiers chrétiens ? Précisément ce que Heidegger appelle leur " être-devenu (Gewordensein) (GA 60, p. 93 ; trad. fr., p. 104) », qui indique l'événement de la conversion. Ce qui importe ici encore, ce ne sont pas tant les circonstances objectives de la conversion (le chemin de Damas pour Paul), mais son vécu ou son expérience (en l'occurrence, la lumière éclatante et la voix), pour autant qu'elle concerne encore les chrétiens dans leur présent : "L'êtredevenu n'est pas un incident quelconque au sein de la vie, mais il est constamment co-expérimenté (miterfahren), de sorte que leur être actuel (jetzig) est leur êtredevenu »(GA 60, p. 93 ; trad. fr. modifiée, p. 106). C'est à la lumière de l'êtredevenu que l'accomplissement de la facticité - cet accomplissement sur lequel se porte une nouvelle forme d'enquête historique - se trouve infléchi dans la direction du hôs mê. Aussi, c'est le faire concret, effectif et actuel lui-même qui est modifié par le négatif : Heidegger parle à ce sujet d'une " différance (eine Retardierung) », d'une rupture dans le cours de la vie facticielle ou encore d'un « être-séparé

21. Ce vocable recevra une attention particulière de la part de Giorgio Agamben, dans cet autre magistral commentaire de saint Paul qu'est Le temps qui reste (trad. par Judith Revel, Paris, Rivages, 2000) et où le modèle de la lecture heideggérienne est tout sauf absent. 
(Abgesondertsein) (GA 60, p. 120 ; trad. fr., p. 135) » qui sont tous les symptômes de quelque chose comme une situation ou une expérience limite.

Ces jalons de l'interprétation phénoménologique des Épîtres de Paul se retrouvent dans l'interprétation que Heidegger donne de saint Augustin. Sa lecture du livre X des Confessions reste en effet gouvernée par la nécessité de retrouver l'accomplissement de la situation historique, mais elle met toutefois en avant une expérience considérablement différente de celle des premiers chrétiens qui a été analysée à partir de Paul : l'axe de cette lecture n'est pas tant l'espérance, la croyance (confiante malgré l'insécurité) dans la parousie, que le danger de la perte de soi auquel se trouve exposé le chrétien. Cette tendance qu'exprime la tentatio augustinienne porte à une acuité particulière l'interrogativité propre à la vie facticielle, car elle ressaisit de façon encore plus tendue le soi vivant sous la figure de la question qu'il se fait à soi-même ( $c f$. GA 60, p. 178 ; trad. fr., p. 197). La problématicité, la difficulté, le fardeau ou le poids d'être (molestia) sont les repères de l'existence exposée à la perte de soi qui guette sous la figure particulière de la tentation. Si cette description ne coïncide pas avec celle de l'expérience des premiers chrétiens, c'est parce que, du converti Paul au converti Augustin, l'accomplissement de la situation historique du phénomène religieux n'est pas le même : le fardeau de la question que le croyant est devenu à lui-même s'est fait de plus en plus lourd (et sa pesanteur s'en trouvera augmentée chez Luther ${ }^{22}$ ).

22. Un autre échantillon important de phénoménologie de la vie religieuse ou d'interprétation phénoménologique d'un texte de théologie est représenté par l'exposé sur Luther que Heidegger fait dans le séminaire du théologien protestant Rudolf Bultmann au cours de sa première année à Marbourg, en février 1924. Dans cette intervention portant sur le problème du péché chez Luther et qui examine successivement la Quaestio de viribus de 1516, la Disputatio contra scholasticam theologiam et la Controverse de Heidelberg de 1518, Heidegger met l'accent sur l'ambition du théologien de proposer une saisie radicale du péché en rapport avec la " corruptio de l'être de l'homme » et de faire ainsi du concept de péché un "concept d'existence (Existenzbegriff)" (infléchissement analogue à celui que subit la tentatio dans la lecture d'Augustin) ( "Das Problem der Sünde bei Luther» (1924), in Rudolf Bultmann/Martin Heidegger, Briefwechsel 1925-1975, éd. par Andreas Großmann et Christof Landmesser, Francfort/M./Tübingen, Klostermann/Mohr Siebeck, 2009, p. 263-27, p. 264 et 267; trad. par Christian Sommer in Le jeune Heidegger (1909-1926). Herméneutique, phénoménologie, théologie, éd. par Sophie-Jan Arrien et Sylvain Camilleri, Paris, Vrin, 2011, p. 259-264, p. 259 et 262). Une vision de la nature humaine en tant qu'originellement corrompue et absolument nécessiteuse du secours de la grâce est solidaire de cette appréhension du péché comme « modalité de l'être de l'homme (Weise des Seins des Menschen)": c'est parce que "la possibilité de son existence ne réside pas en lui (die Möglichkeit seiner Existenz liegt nicht in ihm) » ("Das Problem der Sünde bei Luther» (1924), p. 266 ; trad. fr., p. 260), donc à cause d'une faiblesse qui marque l'accomplissement même de sa vie, que l'homme est par nature disposé envers le pêché. La "présupposition " du "principe du protestantisme " qui est mise au jour dans ce contexte, et dans laquelle on peut identifier à la fois le point de mire de l'approche heideggérienne de la vie religieuse et l'image (fût-elle partielle ou déformée) du Dasein, de l'être-là humain que décrira l'opus magnum de 1927, Etre et temps, se laisse alors expliciter comme suit: " un homme, assis là dans l'angoisse de la mort - dans la crainte et le tremblement, et face aux nombreuses tentations (ein Mensch, der in Todesangst dasitzt - in Furcht und Zittern und viel Anfechtung)» («Das Problem der Sünde bei Luther» (1924), p. 271; trad. fr., p. 264). 
L'ancrage historique de l'expérience chrétienne de la vie et son caractère situé apparaissent ainsi encore une fois comme déterminants dans l'approche des phénomènes de la vie religieuse du jeune Heidegger. Mais l'attention portée à l'historique, sous laquelle on retrouve la marque et l'impulsion de l'herméneutique diltheyenne, s'accompagne également, nous l'avons vu, de l'intention de ressaisir les phénomènes historiques à l'aune de leur accomplissement (Vollzug), par delà leurs expressions objectivées, dans leur dimension d'expérience vécue, et c'est dans l'importance de cette dernière dimension, qui porte l'empreinte de Husserl, que réside le caractère proprement phénoménologique de la démarche. Pour Heidegger toutefois, à la différence de Husserl, la description phénoménologique ne doit jamais mettre entre parenthèses la facticité, c'est-à-dire la teneur concrète de l'existence, pour se concentrer exclusivement ou prioritairement sur l'essence : l'ancrage facticiel reste en effet premier et fondamental. Tous ces points permettent de préciser la manière qu'a Heidegger de procéder en philosophie de la religion et qui conjugue une approche herméneutique attentive au caractère historique de ce qui est décrit voit dans les écrits néo-testamentaires, patristiques et mystiques des expressions historiques de la vie du chrétien, et une approche phénoménologique, elle aussi sensible à la dimension vécue et expérientielle, et en même temps plus soucieuse de la question méthodologique de l'accès à ce qui est à décrire. La synergie de ces approches trouve son expression dans l'idée d'une histoire d'accomplissement (Vollzugsgeschichte), dont l'enjeu est d'assurer une circulation entre le passé et le présent en redonnant une actualité dans le présent aux phénomènes de la vie religieuse appréhendés dans leur caractère historique, pour qu'ils deviennent en quelque sorte contemporains.

\section{Philosophie et théologie}

Pour le projet d'une mise en perspective des apports de la phénoménologie de la vie religieuse du jeune Heidegger qui est ici le nôtre, il importe toutefois hautement de comprendre qu'à leur arrière-fond se trouve une façon spécifique de concevoir le rapport entre philosophie et théologie. Si la reconfiguration de ce rapport était déjà en germe dans l'affirmation déjà examinée de l'athéisme méthodologique de la philosophie, cette thèse recevra une expression plus nette et systématique dans la conférence "Phénoménologie et théologie », prononcée par Heidegger à Marbourg ${ }^{23}$ en juillet 1927 et qu'il nous faut donc prendre en considération ici. Cette conférence insiste en effet sur l'hétérogénéité qui existe entre la théologie comme science positive et historique de la foi et la philosophie ${ }^{24}$

23. Cette conférence a été publiée pour la première fois dans une version bilingue dans les Archives de philosophie, vol. 32, no 3/1969, p. 356-395.

24. Voir aussi la lettre à Elisabeth Blochmann du 8 août 1928, où Heidegger revient sur la question du statut et de la scientificité de la théologie : "La religion est une possibilité fondamentale de l'existence humaine (Religion ist eine Grundmöglichkeit der menschlichen Existenz), même si elle diffère du tout au tout (völlig anderer Art) de la philosophie. Celle-ci à son tour a 
qui apporte un " correctif » ontologique à ses descriptions ontiques (par exemple, par une analyse existentiale de la faute, Schuld, qui éclaire le dogme du péché originel), sans pour autant que la philosophie ait intrinsèquement besoin de se rapporter à la théologie - car par ailleurs, l'idée même d'une " philosophie chrétienne » serait un "cercle carré » (hölzernes Eisen ${ }^{25}$ ) -. Lorsqu'en février 1928, Heidegger reprend à Tübingen la conférence "Phénoménologie et Théologie » sous le titre "Théologie et philosophie ", il laisse davantage transparaître, grâce à ce dernier intitulé, le fait que, par cette conférence, il entend apporter sa contribution à un thème et un sujet à la fois classique et disputé depuis des siècles ${ }^{26}$. L'idée selon laquelle la philosophie apporte un "correctif » ontologique aux descriptions ontiques de la théologie résume sa propre manière de répondre à la question des rapports entre philosophie et théologie. Cette idée est d'autant plus remarquable que, dans l'exposé sur Luther donné en 1924 dans le séminaire de Bultmann (cf. GA 60, p. 178 ; trad. fr., p. 197), Heidegger l'utilisait pour caractériser le protestantisme comme correctif du catholicisme ${ }^{27}$ (et c'était là sans doute aussi un résumé de son expérience personnelle). Or en 1927, cette caractérisation en termes de "correctif » se trouve déplacée et appliquée, de manière significative, aux rapports entre philosophie et théologie. L'introduction de l'idée de correctif passe, dans le contexte de la conférence, par la distinction de l'ontique et de l'ontologique, c'est-à-dire entre ce qui relève des différentes régions d'être et qui fait le domaine des sciences particulières (par exemple, la réalité physique, le psychique, la réalité historique ou sociale, etc.) et ce qui relève de l'être lui-même ou des caractères d'être communs aux différentes régions d'être (ce qui était traditionnellement, pour le dire tout de suite, l'objet de la

elle aussi sa foi - qui est la liberté du Dasein lui-même, telle qu'elle n'accède à l'existence que dans l'être-libre (nur im Freisein existent wird) » (Martin Heidegger/Elisabeth Blochmann, Briefwechsel 1918-1969, éd. par Joachim W. Storck, Marbach am Neckar, Deutsche Schillergesellschaft, 1989, p. 25 ; Correspondance avec Élisabeth Blochman (1918-1969), trad. par Pascal David, Paris, Gallimard, 1996, p. 230-231).

25. "Phänomenologie und Theologie " (1927), in Martin Heidegger Gesamtausgabe, tome 9 : Wegmarken, éd. par Friedrich Wilhelm von Herrmann, Francfort, Klostermann, 1976, $1996^{2}, 2004^{3}$ (noté GA 9), p. 66 ; trad. fr. Archives de philosophie (1969), p. 393.

26. Sur l'histoire des rapports entre philosophie et théologie, voir l'éclairante contribution d'Olivier Boulnois, disponible en ligne sur le site de l'EPHE (http://www.iesr.ephe.sorbonne.fr/ index4493.html), qui souligne bien qu'il s'agit d'une «invention médiévale, le fruit de la rencontre entre la rationalité grecque et la révélation biblique ". Le premier ouvrage proprement dit de théologie au XII ${ }^{\mathrm{e}}$ siècle, la Theologia d'Abélard, règle la question des rapports entre philosophie et théologie en faisant de la théologie l'accomplissement suprême de la philosophie, solution que rend d'une façon quelque peu déformée l'expression latine philosobia ancilla theologiae. À l'égard de cette noble tradition qui reconnaît la dignité de la philosophie sans reculer toutefois à déclarer son infériorité ou sa subordination, Heidegger s'inscrit résolument, comme nous le verrons, en porte-à-faux.

27. Dans l'exposé sur Luther de 1924, Heidegger cite une remarque de Kierkegaard dans son Journal de 1852, dont il résume le sens en identifiant dans le protestantisme le correctif du catholicisme ( Das Problem der Sünde bei Luther » (1924), in Rudolf Bultmann/Martin Heidegger, Briefwechsel 1925-1975, op. cit., p. 271 ; trad. fr., p. 264). 
métaphysique ou encore de l'ontologie, et que Heidegger poursuit sous la forme d'une interrogation renouvelée sur l'être).

Dans la terminologie de Heidegger, la distinction de l'ontologique et de l'ontique, qui permettra de tracer la frontière entre la théologie et la philosophie, recoupe la différence ontologique, à savoir la distinction de l'être et de l'étant. Cela veut dire que, là où la philosophie s'occupe de l'être, la théologie ne s'occupe que de l'étant, et elle serait en cela plus proche finalement des sciences positives que de la philosophie ! C'est là l'une des thèses les plus importantes de la conférence : "La Théologie est une science positive et, comme telle, est absolument différente de la Philosophie " (GA 9, p. 49, trad. fr., p. 359-361). La théologie se caractérise donc par la positivité là où la philosophie se distingue par le caractère ontologique de sa démarche. Mais quel type de positivité ? Heidegger n'ignore certes pas que la révélation, comme base positive de la théologie, n'est pas une réalité factuelle comme la réalité physique ou psychique. À son sol positif, la théologie ne se rapporte pas par l'observation, mais par la foi. Cela ne l'empêche pas d'avoir, aux yeux de Heidegger, sa propre scientificité (remarquons au passage qu'il s'agit là aussi d'un thème classique et disputé : il suffit de penser à l'article 2 de la première question de la Somme théologique de Thomas d'Aquin, qui pose la question de savoir si la doctrine sacrée est une science). Comme science de la foi, la théologie est une science historique, voire historico-pratique, dans la mesure où elle est concernée à la fois par l'historicité de la révélation et par le comportement du croyant. Or, comme le précise Heidegger, « ce n'est pas la foi elle-même, mais la science de la foi, en tant que science positive qui a besoin de la Philosophie »(GA 9, p. 61 ; trad. fr., p. 383), et cela dans la mesure où « tous les concepts théologiques fondamentaux renferment nécessairement en eux la compréhension de l'être » (GA 9, p. 63 ; trad. fr., p. 387). L'exemple pris pour illustrer cette thèse est celui même du concept sur lequel Heidegger portait son attention en 1924, dans son exposé sur Luther au séminaire de Bultmann, à savoir le concept de péché (Sünde). Ce concept recèle une détermination ontologique ou existentiale, c'est-à-dire relative au mode d'être qu'est l'existence, à savoir la faute $(S c h u l d)$. Il ne s'agit pourtant pas de procéder à une déduction rationnelle, mais tout simplement de dégager le soubassement ontologique des concepts que la théologie (chrétienne, en l'occurrence) emploie en les ayant le plus souvent hérités du monde grec ou hébraïque. C'est précisément pour désigner cette intervention clarificatrice de la philosophie que Heidegger parlera ici de correction (Korrektion) et de correctif (Korrektiv) : "L'ontologie ne fonctionne donc que comme un correctif du contenu ontique c'est-à-dire préchrétien des concepts théologiques fondamentaux» (GA 9, p. 64 ; trad. fr., p. 389). Ce geste de correction ne se laisse reconduire ni à une déduction, ni à une fondation : la correction «ne donne bien plutôt qu'une indication formelle, c'est-à-dire que théologiquement le concept ontologique de faute n'est jamais comme tel pris pour thème" (GA 9, p. 65 ; trad. fr., p. 389). Il ne s'agit donc pas de rechercher 
un fondement, mais seulement d'indiquer un soubassement, ou plutôt un horizon ontologique à partir duquel les concepts théologiques sont susceptibles d'être mieux éclairés quant à leurs présuppositions sémantiques et historiques. C'est ainsi qu'il faut comprendre la correction : simplement comme une "indication formelle du domaine ontologique » qui « a pour fonction non pas d'enchaîner, mais inversement de libérer " et d'éclairer (GA 9, p. 65 ; trad. fr., p. 391). En conséquence, le rapport de la philosophie à la théologie peut être précisé comme suit : "La Philosophie est le correctif formel ontologique du contenu ontique, c'està-dire préchrétien des concepts théologiques fondamentaux "; mais en même temps, "la Philosophie peut être ce qu'elle est, sans remplir en fait cette fonction de correctif» (GA 9, p. 66 ; trad. fr., p. 391). Si la théologie, pour s'assurer de sa scientificité, a besoin de la philosophie, cette dernière n'a pas besoin en retour de la théologie et en reste rigoureusement séparée : c'est pourquoi, pour Heidegger, l'idée même de "philosophie chrétienne » est un cercle carré.

On peut supposer cependant que Heidegger n'a pas été tout à fait satisfait de cette manière de résoudre les rapports entre philosophie et théologie. C'est ce que suggère tout d'abord le sort éditorial particulier qu'a eu la conférence "Phénoménologie et théologie », qui n’a été publiée qu'en 1969, dans une version d'emblée bilingue, dans les Archives de philosophie, pour ne paraître en Allemagne chez l'éditeur de Heidegger, Klostermann, qu'en 1970, avec une dédicace à Bultmann. La correspondance de Heidegger avec Bultmann, éditée en 2009, montre en outre à quel point Heidegger a hésité à reprendre et à publier cette conférence, malgré les insistances de son ami et ancien collègue théologien, et ce pour des raisons que l'on pourrait appeler disciplinaires ${ }^{28}$. Ainsi, à l'automne 1928, lorsque Bultmann invite Heidegger à faire partie du comité d'une revue dont il s'apprête à reprendre la publication, la célèbre Theologische Rundschau, et lui propose de publier sa conférence ensemble avec sa propre conférence sur le concept de révélation dans le Nouveau Testament, Heidegger décline; il ne publiera par ailleurs jamais dans cette revue où il figurera cependant comme membre du comité de rédaction de 1929 à 1943. Bultmann aura beau insister en faveur de ce qu'il appelle le «plan initial » (cf. Rudolf Bultmann/Martin

28. Au printemps 1928, au moment où Heidegger apprend sa nomination à Fribourg-enBrisgau qui le fera quitter Marbourg quelques mois plus tard, Bultmann lui écrit qu'il considère son départ comme une perte importante, non seulement d'un point de vue personnel, mais surtout pour la faculté et pour la théologie en général ( $c f$. Rudolf Bultmann/Martin Heidegger, Briefwechsel 1925-1975, op. cit., p. 53). Bultmann n'ignore en effet pas qu'à Friburg il n'y a pas de faculté de théologie protestante, et que donc Heidegger sera replongé, bon gré mal gré, dans « l'élément catholique » (élément qui, notons-le au passage, suscitera de la part de Heidegger, selon divers témoignages d'époque, la plus grande hostilité). Aux regrets exprimés par Bultmann devant la perspective de son départ, Heidegger répond plutôt froidement que plus rien ne le retient à Marbourg, que son intérêt pour la Faculté de théologie a été entièrement dû à son estime et amitié pour Bultmann et que, comme philosophe, il a besoin d'une atmosphère différente, comme celle qu'il a connue à Freiburg et qu'il se réjouit de retrouver ( $c f$. Rudolf Bultmann/ Martin Heidegger, Briefwechsel 1925-1975, op. cit., p. 56). 
Heidegger, Briefwechsel 1925-1975, op. cit., p. 70 et 82) d'une publication conjointe de leurs conférences : il devra finir par accepter de publier la sienne séparément, en 1929, dans la première nouvelle livraison de la Theologische Rundschau.

La Correspondance avec Bultmann montre aussi que Heidegger n'a pas maintenu longtemps la caractérisation ouvertement réductrice de la théologie comme science positive ou discipline ontique qu'il avait proposée dans sa conférence de 1927. Déjà dans une lettre du 23 octobre 1928, il écrit : «Une tentative renouvelée de retravailler ma conférence sur la théologie m'a montré que les frontières ne sont pas encore clairement tracées. Ce qui n'a pas été suffisamment travaillé, c'est le caractère de la théologie, qui la situe au même niveau que la philosophie en ceci qu'elle s'occupe du tout, mais ontiquement »; et par ailleurs, " la philosophie en tant qu'ontologie est elle aussi une ontique entièrement différente d'une science positive ${ }^{29}$ ". La philosophie et la théologie se rejoignent donc par leur visée de la totalité - du tout du monde comme du tout de l'existence - ce qui fait résolument vaciller le partage supposé ferme de l'ontique et de l'ontologique.

Il ne semble toutefois pas que Heidegger ait jamais remis en cause le diagnostic d'une séparation tranchée entre la philosophie et la théologie. En décembre 1930, à l'invitation de Bultmann, Heidegger revient à Marbourg pour donner une conférence intitulée "Philosopher et croire "; il s'agit de fait d'une variante de sa conférence célèbre sur "L'essence de la vérité ", à laquelle il ajoute le paragraphe introductif suivant: «Nous ne parlons pas $d u$ philosopher ou du croire; nous ne parlons pas non plus de leur rapport - mais bien plutôt : nous philosophons, dans la mesure où nous posons la question de l'essence de la vérité. Mais ce faisant, la croyance n'est-elle pas déjà exclue ? Effectivement. Et pourtant nous traitons aussi de la croyance et du rapport à elle - dans la mesure où nous en gardons le silence; le titre [de la conférence] dit que la croyance et le rapport à elle doit demeurer pour la philosophie ce qui est passé sous silence

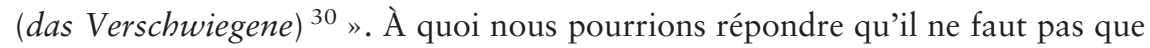
ce qui est passé sous silence demeure, de fait, un impensé.

\section{$*$}

Au terme de cette analyse, plusieurs conclusions concernant la phénoménologie de la vie religieuse du jeune Heidegger s'imposent. Si cette entreprise trouve sa motivation profonde dans la biographie intellectuelle de Heidegger et si elle n'a jamais été reniée en tant que telle, elle ne correspond pas moins à un projet

29. Rudolf Bultmann/Martin Heidegger, Briefwechsel 1925-1975, op. cit., p. 62, nous traduisons ; voir aussi id., p. 87 : « la positivité de la théologie [...] est quelque chose de différent par rapport à celle des sciences ».

30. Rudolf Bultmann/Martin Heidegger, Briefwechsel 1925-1975, op. cit., p. 142-143, note 3 , nous traduisons. 
transitoire qui n'aura pas de vraie postérité dans la pensée de son auteur, rendu de plus en plus soucieux de se démarquer du champ de la théologie et de la philosophie de la religion suite à l'adoption précoce de la thèse d'un athéisme méthodologique de la philosophie : nous avons pu le constater en examinant le diagnostic sur les rapports entre philosophie et théologie auquel aboutit l'importante conférence de 1927. Néanmoins, cela n'enlève rien à l'intérêt et à la force de la démarche d'interprétation phénoménologique qui s'illustre dans les cours de 1920-21 et de 1921, démarche qu'il serait donc possible d'autonomiser et de prolonger, et dont l'attention à l'expérience vécue et à l'accomplissement de la situation historique du croyant fournit assurément le principe d'un renouvellement méthodologique qui pourrait être d'actualité encore aujourd'hui.

\section{Claudia SERBAN \\ Fondation Thiers/CNRS (Archives Husserl) Claudia-Cristina.Serban@ens.fr}

\section{La Phénoménologie de la vie religieuse du jeune Heidegger : une mise en perspective}

Grâce à sa double filiation husserlienne et diltheyenne, le projet heideggérien d'une phénoménologie de la vie religieuse s'avère porteur d'un véritable renouveau méthodologique. L'attention portée à la dimension historique s'accompagne en effet de l'intention de ressaisir les phénomènes étudiés à l'aune de leur accomplissement (Vollzug), dans leur dimension d'expérience vécue, par delà leurs expressions objectivées. Cependant, la position de Heidegger à l'égard de la philosophie de la religion est marquée par plusieurs tensions, dont notamment celle entre son auto-caractérisation comme "théo-logien" chrétien et son plaidoyer en faveur d'un athéisme méthodologique. Cette tension conduit à affirmer une nette hétérogénéité entre théologie et philosophie, selon le partage de l'ontique et de l'ontologique. L'examen de la correspondance de Heidegger avec le théologien protestant Rudolf Bultmann invite toutefois à nuancer ce diagnostic.

Mots-clés: Heidegger, phénoménologie, théologie, religion, philosophie.

\section{Young Heidegger's Phenomenology of Religious Life: Broadening the Perspective}

Thanks to its dual filiation, Husserlian and Diltheyan, the Heideggerian project of a phenomenology of religious life proves itself to be rich of a genuine methodological renewal. The attention to the historical dimension is accompanied by a reassessment of the phenomena in the light of their accomplishment (Vollzug), as lived experiences, beyond their objectivized expressions. However, Heidegger's position towards the Philosophy of Religion is marked by several tensions, amongst which the one between his self-characterization as a Christian "theologian" and his plea in favor of a methodological atheism. This tension leads to the statement of a heterogeneity between 
Theology and Philosophy, following the division between the ontic and the ontological. Heidegger's Correspondence with the protestant theologian Rudolf Bultmann hints nevertheless at the reasons for which such a diagnosis cannot be maintained.

Key words: Heidegger, Phenomenology, Theology, Religion, Philosophy.

\section{La Fenomenología de la vida religiosa del joven Heidegger : una puesta en perspectiva}

Gracias a su doble filiación husserliana y diltheiana, el proyecto heideggeriano de una fenomenología de la vida religiosa se revela portador de una verdadera renovación metodológica. La atención llevada a la dimensión histórica se acompaña en efecto de la intención de retomar los fenómenos estudiados en el momento de su cumplimiento (Vollzug), en su dimensión de experiencia vivida, más allá de sus expresiones objetivadas. Sin embargo, la posición de Heidegger respecto de la filosofía de la religión está marcada por varias tensiones, especialmente aquella entre su auto-caracterización como "teo-logo" cristiano y su posición a favor del ateísmo metodológico. Esta tensión conduce a afirmar una clara heterogeneidad entre teología y filosofía, según la división entre la óntica y la ontológica. El análisis de la correspondencia de Heidegger con el teólogo protestante Rudolf Bultmann invita a matizar este diagnóstico.

Palabras clave: Heidegger, fenomenología, teología, religión, filosofía. 